\title{
Rendre invisible le visible : les sculptures de Jorge Iglesias
}

Antonia GARCIA CASTRO

\section{(2) OpenEdition \\ 1 Journals}

\section{Édition électronique}

URL : http://journals.openedition.org/conflits/1838

DOI : $10.4000 /$ conflits.1838

ISSN : $1777-5345$

Éditeur :

CCLS - Centre d'études sur les conflits lilberté et sécurité, L'Harmattan

\section{Édition imprimée}

Date de publication : 1 juin 2005

Pagination : 207-209

ISBN : 2-7475-8911-0

ISSN : 1157-996X

\section{Référence électronique}

Antonia GARCIA CASTRO, « Rendre invisible le visible : les sculptures de Jorge Iglesias », Cultures \& Conflits [En ligne], 58 | été 2005, mis en ligne le 10 octobre 2005, consulté le 30 mars 2021. URL:

http://journals.openedition.org/conflits/1838; DOI : https://doi.org/10.4000/conflits. 1838

Ce document a été généré automatiquement le 30 mars 2021.

Creative Commons License 


\title{
Rendre invisible le visible : les sculptures de Jorge Iglesias
}

\author{
Antonia GARCIA CASTRO
}

1 Le dispositif est une sorte de toute petite scène éclairée par deux ampoules électriques. L'une côté cour. L'autre côté jardin. Côté cour, un vase. On le dirait ancien. Couleur ocre. Tout comme la toile de fond. Rien d'autre. Soudain, une main fait son entrée, va vers le milieu de la scène; un des doigts semble se glisser dans quelque chose. Dans quoi ? On ne voit que le fond ocre. Pourtant, le bout du doigt disparait. Puis, réapparaît. Très lentement la main saisit le vase ancien et le transporte vers ce même point situé au milieu de la scène : le vase est couché, comme suspendu dans l'air. La main ramène le vase à sa place avant de se diriger vers l'une des ampoules : elle en masque en partie la lumière. C'est alors qu'on la voit. Le contour apparaît clairement sous l'effet de cette lumière diffractée : c'est le contour d'une bouteille, ocre elle aussi, située au milieu de la petite scène. Visible seulement si l'une des ampoules est masquée. Invisible sous trop de lumière.

2 On « comprend » que le vase n'était pas suspendu mais tenu en équilibre sur un support momentanément rendu invisible. Mais le vase suspendu : on l'a « vu »...

3 Telles sont les sculptures de Jorge Iglesias, plasticien argentin. Elles se jouent de nos perceptions. Elles jonglent avec nos possibles et impossibles. Celle-ci porte le nom de «Cocafull ». On peut aussi se rapporter à "Copa Rosa », « Vela », « Obelisco Invisible »" Le plasticien en donne pour définition: «Objets que l'on peut voir sous certaines conditions de lumière et pas sous d'autres ".

4 Le caractère inédit du dispositif est précisément dans cette exposition : « les objets sont invisibles en pleine lumière et directement soumis à la vision du spectateur ». L'enjeu pour l'artiste? Il y revient dans un entretien dont nous reproduisons ci-dessous quelques extraits ${ }^{2}$.

Utiliser l'invisibilité au service d'une nouvelle visibilité

5 « Un jour, alors que je me trouvais face à mon modèle (je peignais alors sur une toile), je me suis dit que si les raisons pour lesquelles je le voyais étaient celles que je pensais, il était possible de le rendre invisible. Que si je voyais cet objet en raison des différences 
des tons clairs et obscurs et des diverses couleurs en présence, le fait de peindre le contraire sur l'objet même annulerait tout ce qui me permettait de le voir. Peindre pour ainsi dire « à contrario » ce serait comme de peindre un négatif - à la manière du photographe - sur le corps même et avec une précision capable d'annuler le visible (...).

6 - A votre avis pourquoi cette idée n'a-t-elle pas été formulée auparavant par d'autres artistes?

7 - Une seule explication me vient à l'esprit : bien que les conditions intellectuelles et physiques aient été données, il n'en allait pas de même pour les conditions psychiques ; le mandat culturel en sens inverse était si fort qu'il empêchait l'idée de surgir.

- Et quel était ce mandat culturel?

9 - Que le peintre et le sculpteur n'ont eu de cesse de peindre et de sculpter afin de rendre des choses visibles (...). Ainsi, si j'avais installé un robinet sur un mur et demandé à un peintre de "faire son travail », sans donner aucune autre explication, il est certain que ce dernier aurait peint sur le mur un robinet semblable pour me faire croire qu'il y en avait deux : l'idée ne lui serait pas venue de peindre sur le seul robinet existant pour faire en sorte qu'il n'y en ait plus aucun. Ce mandat vaut également pour le peintre non réaliste. Paul Klee, par exemple, disait qu'il voulait rendre visible l'invisible, mais il n'a jamais dit qu'il voulait rendre invisible le visible. Pour une raison que j'ignore ce mandat culturel s'est rompu en moi (...).

10 Je me souviens que lorsque j'étais étudiant nous fréquentions un café où il y avait un graffiti sur le mur. Il disait: "Un trou dans le néant ». Cette phrase a déchaîné une série de discussions. Les écrivains nous disaient que là, justement, se trouvait notre limite. Car eux pouvaient écrire des phrases poétiques de ce type mais que nous, plasticiens, nous ne pourrions jamais les représenter : un sculpteur ne pouvait que faire un trou dans un corps, jamais dans le vide. Les écrivains discutaient également entre eux. Les plus rationnels soutenaient que la phrase en question était valable pour une poésie surréaliste mais pas en philosophie, par exemple. Parce que l'homme invente des mots pour définir des choses - tels que trou et néant - mais s'il ne prend pas garde au moment de les combiner, il peut générer des phrases contradictoires comme celle-ci qui, au fond, ne signifient rien.

11 Me rappelant tout cela, et disposant désormais de la possibilité de rendre invisibles des objets, j'ai eu l'idée de réaliser un corps (un cube dans ce cas), de lui faire un trou et de peindre ensuite - depuis les bords de ce trou en allant vers l'extérieur - en rendant tout le reste invisible. Le résultat perceptuel fut celui d'un trou flottant dans le vide (...). Désormais le fait de dire « un trou dans le néant » n'était plus une contradiction mais une phrase aussi descriptive que « un champ vert » ou « un ciel bleu » puisqu'elle ne faisait que décrire un fait perceptuel.

12 Un autre enjeu était d'utiliser l'invisibilité au service d'une nouvelle visibilité. On peut laisser flotter des objets en rendant invisible le corps qui les soutient, ou faire couler de l'eau du néant en rendant invisible le robinet... On peut aussi faire bouger des objets inanimés. Par exemple, en réalisant une sculpture avec plusieurs bras invisibles et en les rendant visibles un par un, cela donnerait l'impression d'un seul et même bras en mouvement ». 


\section{NOTES}

1.. Ces œuvres - entre autres - sont exposées sur le site internet de l'artiste, classées par genre : on les découvre sous forme de très courts vidéo-clips permettant de saisir les jeux de lumière. L'Obélisque Invisible - de trois mètres de hauteur - est particulièrement étonnant. Cette œuvre fut partie prenante d'une chorégraphie ( «uenos Aires Luz »). Sur la scène - une très grande scène - des danseurs évoluent. L'un d'eux se détache. C'est une danseuse. Elle est suspendue. Les autres lèvent les yeux et suivent ses mouvements. Soudain, elle se glisse derrière une figure triangulaire que l'on n'avait pas encore vue - que l'on ne pouvait pas encore voir : elle l'entoure de ses bras et poursuit sa danse dans les airs. Sur cette grande scène, la toile de fond c'est le corps en mouvement de la danseuse : sur les pans sombres de sa robe se dessine la pointe d'un Obélisque blanc. Voir http://www.jorgeiglesias.com.ar - Les informations communiquées dans cette brève présentation - en particulier les propos du plasticien sont issues de ce site.

2.. Entretien réalisé par J.R. Rodríguez, intégralement reproduit (en espagnol) sur le site déjà cité. Outre les enjeux de l'invisibilité appliquée aux sculptures, le plasticien y aborde d'autres recherches relatives à l'usage de la lumière notamment en peinture. Notons, au sujet de ces recherches, qu'en 1995 le Sénat de la République Argentine a déclaré ces recherches « d'intérêt culturel ».

\section{INDEX}

Mots-clés : art, regards sur l'entre-deux, visuel

\section{AUTEUR}

\section{ANTONIA GARCIA CASTRO}

Antonia Garcia Castro est docteur en sociologie, co-rédactrice en chef de la revue Cultures \& Conflits. 